\author{
EVS27 \\ Barcelona, Spain, November 17-20, 2013
}

\title{
Modeling and thermal simulation of a PHEV battery module with cylindrical LFP cells
}

\author{
Cicconi P. ${ }^{1}$, Germani M., Landi D. \\ ${ }^{1}$ Università Politecnica delle Marche, via Brecce Bianche,60131-Ancona-Italy,p.cicconi@univpm.it
}

\begin{abstract}
Generally a part of electric vehicle diffusion is still based on marketing of cars and vans suitable for specific use like work vehicles. A flexible design methodology is required to support rapid prototyping and product customization in the market of tailored EV/PHEV. The research focuses the cooling simulation for a PHEV Li-Ion battery. The thermal analysis is based on the physical parameters of the single cell and on the experimental data. The proposed methodology concerns firstly an analytical approach which evaluates the average heat generated by a single cell during working condition. Then the proposed virtual prototyping analysis has been divided into two levels: the thermal simulation of one cell, and the CFD analysis of a battery module. This workflow has been applied to support the design of a battery pack for a prototypal ecological hybrid vehicle. That test case vehicle is a small van, used for the curbside collection, which has in parallel an internal combustion engine and an electric motor supplied by a LFP battery with small cylindrical cells. The analysis concerns one of the four module which constitutes the whole battery pack. The virtual model has been parameterized and the behavior of air cooling system has been evaluated through virtual tools.
\end{abstract}

Keywords: lithium battery, cooling, modeling, simulation, PHEV

\section{Introduction}

Nowadays, the development and use of electric vehicles are strongly encouraged to reduce the environmental impact of transport issues. Apart from large companies, which tend to develop general purpose solutions, small and medium sized companies aim to produce customized lots of vehicles for specific customer needs (i.e. municipalities). In this case, the design of vehicles and sub-systems has to be rapid and sufficiently flexible in order to analyze the specific applicative context and translate it into a successful solution [1]. Simulation and early testing are essential phases to save time and avoid many prototyping iterations.

Generally, the Virtual Prototyping (VP) techniques are widespread in engineering design. These kinds of solutions, which are suitable for large companies, have to be customized for use in producing small lots [2]. In particular, in the context of PHEV, these tools are used to analyze different issues, such as the co-simulation of the real driving cycle, the computational fluid dynamic (CFD) study of the cooling effect, and the computing of the electrochemical formulas to estimate the voltage curves and the heat generated at different current rates. A particular limit is present in the battery cooling analysis where commercial tools provide an electrochemical 
characterization which requires a deep knowledge of the many parameters related to the cell materials. The issue concerns the lack of knowledge related to the compositions and dimensions of the internal cell material layers.

The proposed approach is outlined in collaboration between an Italian company which elaborates and markets medium-small ecological vehicles powered by natural gas, electricity or hybrid powertrain (gasoline and electric in parallel mode). This small-medium enterprise works in the industry of customized items, where the lead motive is the reducing of time and cost. The paradigm is the just in time (JIT) production which requires flexible and agile tools to support the designing and manufacturing processes. In the design of customized vehicle is very important to get smart design tools and methods to support engineers in their tasks.

Next sections explain our research work on the thermal issue for the automotive battery pack. The proposed methodology provides an analytical valuation of heat generated by each $\mathrm{Li}$ ion cell and the integration of this calculation in the virtual prototyping analysis based on FVM (Finite Volume Methods) tools. A test case has been exposed to explain the defined workflow in the cooling design for PHEV battery pack. The cells used in the proposed case are LFP cylinders suitable for an hybrid application.

\section{Background research battery design}

on

Every rechargeable lithium ion (Li-ion) battery is constituted by a packing of cells (also known as battery pack) so that the final electric power is the sum of all single elements [3].

The process of Li-ion battery design concerns modeling and simulation regarding whole battery pack. In industry, the actual strategies for battery pack design are mainly related to the market size. The market of large-scale production requires reliable batteries for standard applications. Conversely small productions, with a little volume of customized units, focuses the satisfaction of particular customer specifications coupled to a reduced time-to-market. On the one hand, large battery manufacturers can use expensive design tools and subdivide additional costs on a large number of sales. On the other hand, small and medium enterprises have to increase the own knowledge and formalize the rules of expert designers in low-cost customized tools, in order to contain excessive costs. The proposed research activities is focalized on customized production of a typical European SME (Small Medium Enterprise).

The traditional design approach, commonly used in many SMEs, is mostly based on the experience of engineers. So that designers use basic calculation tools to analyse and elaborate new solutions. The engineer calculates the electrical layout configuration, determines the geometrical shapes and defines the cooling system size. Then, the corresponding physical prototype is manufactured and physical tests give him/her the performance quality. Possible design errors introduce expensive iterations, thus time to market gets longer. The main activities are repetitive tasks and designers are limited to use own experience on past project data (such as empirical tables, drawings, reports and feedbacks). The main parameters in battery designing are: the operative conditions, the single cell type, the cell number, the battery layout, and finally the evaluation of heat released by the electrochemical reactions.

According to the temperature problems in Li-ion cells, a battery pack needs successful thermal energy management and a cooling system to favor heat dissipation and thermal runaway. The two required aspects for an automotive lithium ion battery are: an optimum operating temperature range and small temperature variations. Some researchers have analyzed methods to optimize the geometrical and fluid dynamic parameters in cooling design [4].

The proposed research aims to define a methodology suitable to evaluate different kinds of cooling systems. The objective is to develop a workflow for battery pack thermo-fluid simulation, where the electrochemical heat source is evaluated by an analytical calculation. The main difference with the state of the art is the development of a spreadsheet for rapidly computing thermal power values at different rates of charge and discharge. The second difference is the application of the analytical thermal power values, previous calculated, into the VP tools for the cooling fluid dynamic analysis.

\subsection{Thermal issue}

In Li-ion battery cells the temperature influences: electrochemical reactions, round trip efficiency, charge acceptance, power and energy capability, reliability, life cycle and cost [5]. Furthermore, in electric vehicles, battery temperature increases during discharge with a huge peak for high current rates. 
Generally an excessive temperature degrades performance and a limited thermal dissipation can even produce burning in Li-ion battery cells. Great importance is also given to battery safety concerning heat accumulation due to the lithium flammability and explosiveness. The temperature raises two very important issues in $\mathrm{Li}$-ion batteries: safety risks and material aging. The safety risks are lead to high temperature values during discharge over the permitted level. The material aging are related to the operative cycles and to the achieved temperature levels during working conditions. Moreover, in Li-ion battery pack is also very important the temperature distribution. A non-uniformity in temperature of a cell pack favors localized deterioration in single battery elements, this introduce efficiency losses (as ohmic resistance) and lifespan reduction in whole pack [6].

The thermal model characterization for a $\mathrm{Li}$-ion cell is important to predict the average temperature at different current rate in the early design phase. The explained thermal analysis is based on a study by Thomas and Newman [7] which evaluates the heat produced by the single cell. In particular, a simplified form of Newman formula (1) has been considered in an analytical approach to estimate the heat source at different current rate for each lithium battery cell.

$\dot{Q}=I\left(V-E_{0}\right)-I T \frac{\partial E_{0}}{\partial T}$

In Eq. (1) the term $I$ indicates the instant current value, the term $V$ indicates the related electrical potential (voltage measured at the cell poles), while $E_{0}$ is the relative open circuit voltage, and $T$ indicates the temperature value.

\subsection{Battery simulation}

Several research works have analyzed the issue of simulation for lithium-ion battery packs. That mainly concerns the electrochemical and thermal cell characterization. Particularly the electrical aspect of lithium-ion cells regards the property to generate a potential difference between negative and positive pole (voltage), while the chemical aspect concerns the capacity to move quantity of electrons and ions through each battery sublayers. The thermal behavior of Li-ion cell is related to the heat dissipated from electrochemical reactions inside single battery element.

Thus electrochemical and thermal analysis are coupled features in simulation virtual analysis. In literature several researchers have investigated only the electrochemical behavior. However those, who have analyzed the thermal analysis, have also investigated the electrical model characterization. Below an explanation regarding recent works on battery simulation.

The electrochemical simulations on Li-Ion batteries concern the evaluation of voltage curves and state of charge (SOC) at different current rates in charging and discharging [8]. Often, in electrochemical simulations, the input current profile wants really reproduce the effective driving cycles load for electric vehicles. This type of simulation supports the engineer in modeling of tailored electrical layout configuration for batteries This type of simulations supports the engineer in modeling of tailored electrical layout for batteries. Generally equivalent circuit describes the electrical behavior of the cell system as an approximation of non-linear real phenomena. Therefore conventional linear circuit [9] allows the engineer to predict a reliable voltage over poles with simple component like an RC filter (resistor-capacitor) and an ohmic resistance. The calculation of real voltage curves gives an important feedback regarding the battery performance in terms of energy and power analysis. In addition, the SOC valuation is an important feature to predict the battery autonomy [5].

On the other hand, the thermal simulation regards the heat generated by the electrochemical reactions and also analyzes the cooling performance. Many simulation techniques have been involved in literature. A development of Genetic Algorithm has been extended to optimizing the NTU (Number of Transfer Unit) model in lithium cells for heat exchange study [10]. This approach proposes good results, but the application is often customized to specific cooling system, as i.e. in [10] where the heat exchange is encouraged by tubes with cool air. Using a Genetic Algorithm approach is possible to achieve good results but it is not possible to define a routine common for different battery types and models.

There are several types of thermal simulation models based on: lamped element model, finite element analysis (FEA) and computational fluid dynamics (CFD) analysis with finite volume methods (FVM) [11]. Generally the lamped element model approach provides simple thermal models in lithium battery cell [12], but this requires the definition of several parameters through experimental measurements or technical datasheet. These technical specifications on cell sub-layers are often not provided by manufacturer, 
so it is fundamental the planning of useful experiments to achieve the necessary data.

Actually there are reliable commercial tools for battery modeling, but cost and complexity of these tools limits their diffusion. So that several researchers have analyzed customized tools for specific cells and battery pack layout. However, rapid tools are necessary to support engineer for evaluating thermal battery behavior inside the European SMEs that are working in automotive industry. This tools have to provide a common workflow design for several lithium-ion battery cases.

\section{The framework methodology}

For the proposed research it has been formalized a modeling workflow to support the engineer during the early design phase. The research aim is not only battery modeling, but also the reduction of lead time into a typical SME. The target is the design of cooling layout for a battery pack in an automotive powertrain. As cited before, a reproducible methodology is very accepted to support the design of customizable products in SMEs.

In particular Figure 1 describes the main framework platform which has been followed for the test case proposed in next sections. The scheme presents four different modules: HD tools, KBE, DB and VP tools. The first module regards the hardware tests of a single cell at electrical test bench for data gathering. The voltage curves and the open circuit voltage behavior are the main data which are analyzed during the cell electrical test.

The KBE module represents the knowledge based engineering tools which support the designer in his tasks. Specifically the KBE application is a special type of knowledge based system which has an important focus on product engineering design and downstream activities such as analysis, manufacturing, cost estimation and even sales. But in our approach it has been analyzed only the phase regarding the product design and performance analysis. The KBE tool is composed by the Test Configuration form where the designer defines the electrical test for each type of cell. This level is connected to a database of models which can be selected by the designer through a filtering scheme. Next level in KBE tools is represented by the First Analytical Thermal Analysis, which is constituted by a parametrical spreadsheet for evaluating heat source. Using this tool, the designer can introduce an electrical current profile and then obtain the thermal power levels as a curve over time. The level of Pack Layout Configuration is connected to the CAD system tool and to a collection of template models. This third level supports the engineer in the geometrical layout definition and in the assembly of all battery cell arrays in a 3D model geometry. Generally, development of $\mathrm{KBE}$ applications involves a reduced time of product configuration phase, aids in decision-making activities and automates repetitive procedures. In this paper has been explained the proposed platform architecture, but the KBE tools has not been very detailed in order to give more relevance to the thermal analysis and virtual prototyping in battery modeling.

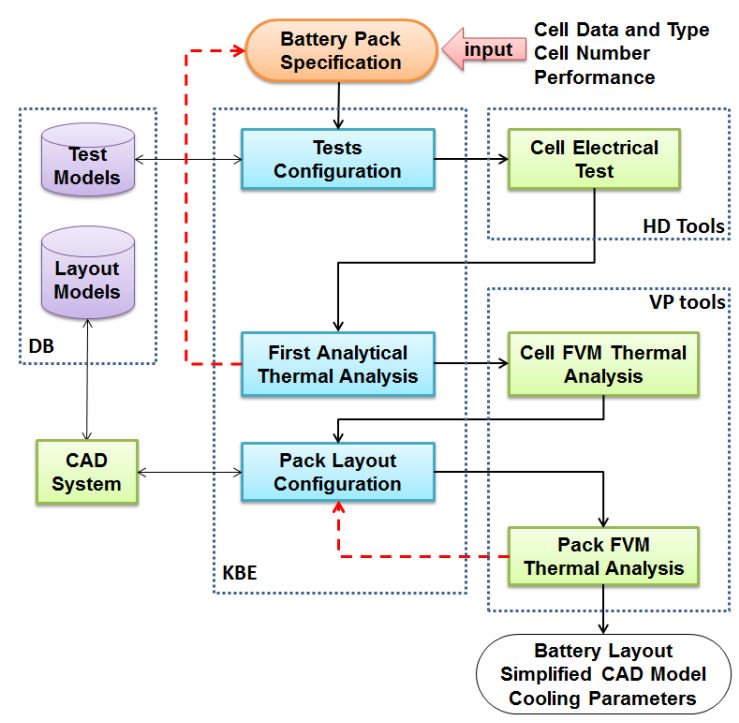

Figure 1: The scheme of proposed methodology

The proposed VP tools module concerns the virtual prototyping simulations for battery modeling, and also the stand alone module of CAD system. Particularly the virtual prototyping analysis regards two levels: the Cell FVM thermal analysis, and the Pack FVM Thermal Analysis (where FVM means Finite Volume Methods). The first level investigates the heat distribution in the virtual model for a single Li-ion cell. The boundary condition is given by the input thermal power profile calculated in the analytical thermal analysis using the KBE tool. While the second level concerns the virtual simulation of thermal and cooling behavior in complete battery pack model. In particular this type of simulation regards two aspects: the CFD analysis of fluid cooling flow, and the thermal analysis of each elementary cell in order to investigate the temperature distribution. For lithium-ion batteries is recommended a constant working temperature with a maximum difference of $2-3^{\circ} \mathrm{C}$ between 
minus and maximum values. Thus the cooling validation concerns also the verification of temperature distribution, in order to guarantee an uniformity in heat exchange. Using CFD tools it is possible to set parameters in virtual model such as cooling temperature, and fluid flow velocity, while the geometrical parametric variation is related to the $\mathrm{CAD}$ model.

The connection between each platform module are symbolized in Figure 1, where dashed line means that the design workflow can turn back in presence of failure settings. The platform provides the First Analytical Thermal Analysis tool to early valuate the thermal behavior of one cell. This analytical tool carries out a thermal characterization based on previous experimental tests (HD tools). The heat source values are calculated by an analytical formula and depend on the cell's physical parameters, current rates and time period. This early analysis mainly considers a one battery cell working in natural convection in order to reproduce the test bench conditions in virtual environment. But it is also possible to calculate the cell heat exchange in forced convection by analytical formulation.

Input data for the whole proposed workflow is constitute by the cell details such as type, electrical capacity, energy and power density, heat capacity, thermal conductivity. Other input is the electric layout of battery pack: cell count, electrical connection, etc.

Output data are the geometrical battery layout, a simplified battery model, a maps of a temperature distribution, and the definition of cooling parameters.

\section{Battery modeling for PHEV}

An hybrid application has been chosen to explain the proposed methodological approach. In particular a prototype Li-ion battery module for a PHEV has been analyzed through virtual tools. The related vehicle, which is designed for the European market, is a prototype van for curbside collection. This special vehicle is configurable on the customer's requirement and has an hybrid propulsion with gasoline engine and electric motor, which are coupled in mild-parallel mode with regenerative braking. The internal combustion engine is suitable for extra-urban distances at constant velocity, while the electric powertrain is recommendable for the urban startand-stop paths at slow velocity. Furthermore, using the energy recovery brake is possible to recharge a part of Li-ion battery during the journey routes. This prototypal hybrid vehicle presents a single $25 \mathrm{~kW}$ drive electric motor with a $7.55 \mathrm{~kW}$ lithium-ion battery pack; while the internal combustion engine has 4 cylinders and a $1.2 \mathrm{~L}$ in capacity. The battery size focuses the hybrid aspect of the prototype vehicle. This vehicle can work in electric mode during the short paths around buildings, and use the internal combustion engine crossing the city districts. The max vehicle velocity is $70 \mathrm{~km} / \mathrm{h}$ if gasoline fueled, while 50 $\mathrm{km} / \mathrm{h}$ if electric powered.

In details, the prototypal battery model provides 212 LFP 10 Ah cylindrical cells (Table 1), separated in 4 module of 59 cell each one. The use of a low capacity cell is explainable by the technical characteristics suitable for a prototype hybrid vehicle. The selected cell has a different application range due to its cylindrical geometry, which is suitable for packaging, and due to the high discharge current rate of $3 \mathrm{C}$ in continuous and $10 \mathrm{C}$ in peak. Thus, this Li-ion cell type is able to be adopted in hybrid powertrain application, where battery provides support in starting, parking and driving for short paths. It is not required an important fixed kilometers range for hybrid vehicle. Farther, the proposed application requires slow velocity in main working condition for curbside collection. Besides, the chosen cells can be rapidly charged at least $2 \mathrm{C}$ rate, instead of the usual limit of $0.5 \mathrm{C}$ or $1 \mathrm{C}$.

Table 1 Technical data sheet of LFP cell 10 Ah

\begin{tabular}{|l|l|}
\hline Chemistry & LFP $(\mathrm{LiFePO} 4)$ \\
\hline Nominal Voltage & $3.2 \mathrm{~V}$ \\
\hline Geometry & Cylinder \\
\hline Nominal Capacity & $10 \mathrm{Ah}$ \\
\hline Max Discharge & $3 \mathrm{C}(30 \mathrm{Ah})$ \\
\hline Max Charge & $2 \mathrm{C}(20 \mathrm{Ah})$ \\
\hline Weight & $330 \mathrm{~g}$ \\
\hline
\end{tabular}

Each battery module is air-cooled by two compact fan wheels, and the geometry layout has been defined using virtual tools and physical experiments on the selected cells.

Next section explains the thermal model characterization for one cell using the analytical analysis of heat source. The design methodology has been applied to the modeling and simulation of proposed Li-ion battery pack. Then, the virtual temperature distribution has been analyzed at FVM tool. Afterwards, the research work proposes the geometrical parameterization of battery pack and the CFD simulation for a defined model of 59 LFP cells. 


\subsection{Thermal model}

The proposed thermal model solves Eq. (1) to evaluate the electrochemical heat source during charge and discharge. The geometry domain analyzed is the cell cylindrical volume. The aim of the analytical thermal model is to provide a tool to aid the designer in the estimation of the max heat generated during the working condition. The input is a current profile which reproduces the electrical load during the standard operative phases. Instead the required physical parameters, related to cell type and chemistry, are recovered from database or gathered from experimental tests. These physical parameters are: the OCV (open circuit voltage), indicated as the term $E_{0}$ in Eq. (1), and the variation of $\mathrm{OCV}$ in function of temperature $\left(\partial \mathrm{E}_{\mathrm{o}} / \partial \mathrm{T}\right)$. Test for evaluating OCV curves concerns discharge steps of $10 \% \mathrm{SOC}$ at $1 / 3 \mathrm{C}$ current rate. While the study of temperature effect over OCV are analyzed by the maintaining of cell at controlled temperature in a climatic chamber. In Figure 2 is shown the trend of OCV acquired through experimental tests at several SOC steps.

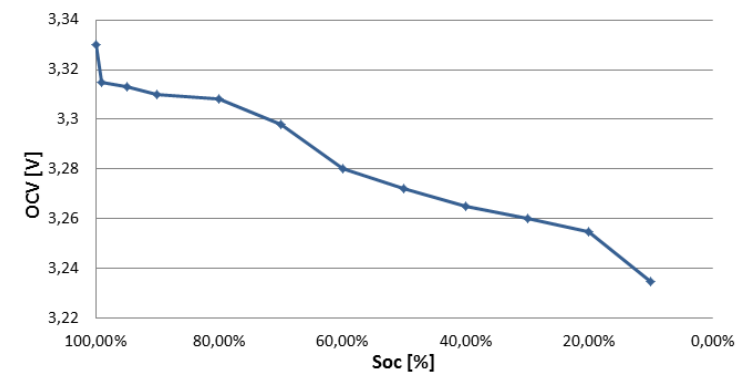

Figure $2 \mathrm{OCV}$ trend for selected cell type

The operative condition, chosen for the proposed test case, is the thermal load related to one cell during a driving path powered by electrical motor. In particular, as a standard run, it has been chosen a path constituted by four repeated ECE R15 Urban Driving Cycles (Figure 3). The standardized ECE cycle reproduces the typical driving conditions of busy European cities, characterized by low engine load, and a maximum speed of $50 \mathrm{~km} / \mathrm{h}$. This kind of driving cycle is suitable to reproduce the real operating condition introduced for this test case, even if in the last years several researches have promoted alternative cycles more realistic.

Figure 4 describes the electrical load on one cell into the prototype battery pack. The current profile related to the selected driving path has been calculated considering the electrical pack configuration and the vehicle dynamic behavior.
Then an experimental test has been conducted, applying this current profile on one cylinder cell, in order to acquire the voltage curve related to the defined driving cycle.

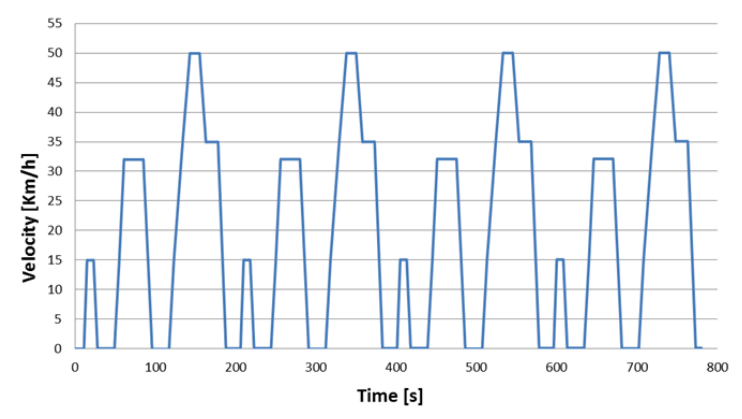

Figure 3 ECE R15 Urban Driving Cycle repeated 4 times (780 s)

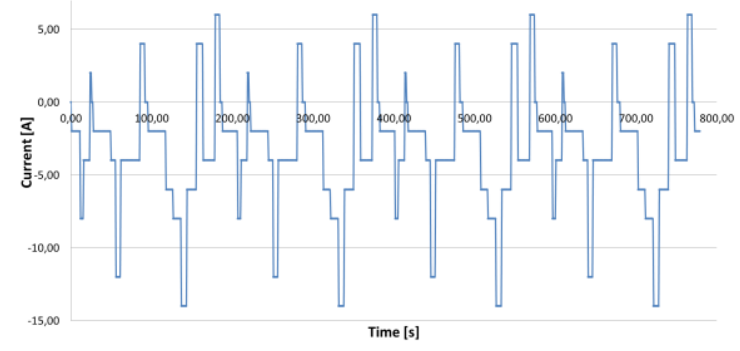

Figure 4 Graphic of current profile for one cell under the driving cycle analysed.

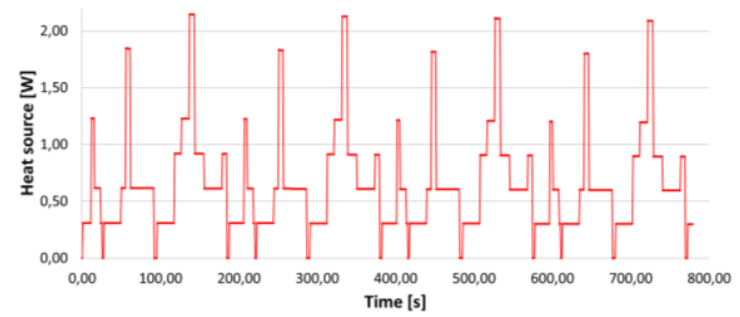

Figure 5 Graphic of the analytical calculation of heat source generated by electrochemical reaction for one cell under load condition of four repetitive ECE R15 driving cycles.

The graphic in Figure 5 shows the variation of heat generated during the driving cycle, in particular this profile has been calculated resolving the analytical formula to evaluate the heat source released by main electrochemical reactions.

The follow section reports how the cell thermal profile can be reproduced through the numerical formulation of the virtual simulation, in order to analyze the maximum average temperature achievable under operative condition. While the analytical profile of heat generated becomes an input for the simulation analysis. 


\subsection{Cell simulation}

The methodological approach includes two levels of virtual simulation: the one cell thermal analysis, and the CFD simulation of a battery pack. This section explains and describes the thermal simulation approach for one cylindrical LFP cell of $10 \mathrm{Ah}$ in capacity. The target of these simulations is to rapidly valuate the cell temperature level using virtual tools. Particularly the application case concerns a battery air cooled. The main input, in this valuation, is the heat generation rate which is time dependent. The values of thermal power are calculated by the analytical tool as cited before.

The virtual boundary condition reproduces the thermal generation in operative condition for one cell. At this step it has been analyzed the natural convective heat exchange, so that it has been possible to reproduce the temperature profile during test condition inside a climatic chamber. The convective heat transfer coefficient has been evaluated by empirical formulations for natural convection.

The profile of heat generation, during time, has been set according to the analytical calculation described in previous phase. As cited before, it has been reproduced the thermal load due to a path of four repeated ECE R15 Urban Driving Cycles. In Figure 6 the temperature profile elaborated by an thermal FVM analysis in comparison with the real temperature trend during test, at the defined working condition.

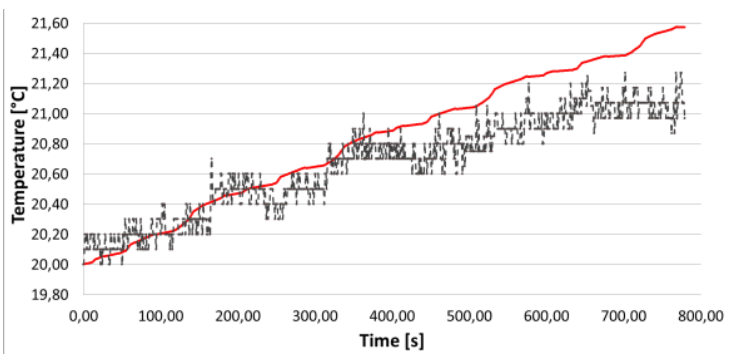

Figure 6 Comparison between simulated temperature (solid red line) and real values monitored by IR-

Camera (dark dotted line) for one single cell in natural convection during the defined driving cycles.

The red solid line (Figure 6) describes the simulated average temperature profile for the analyzed battery cell (cylindrical LFP $10 \mathrm{Ah}$ ) under the operating condition related to the defined driving cycle. While the dotted line represents the real temperature values for one cell monitored by IR camera at test bench inside the climatic chamber in natural convection. The average error for simulation values is almost $5 \%$ compared with the experimental values. This result is acceptable, however it should be considered that the simulation is limited to a transitory of only 13 minutes and the heat outlet is no so high during standard ECER15 driving cycles. The max temperature estimated is almost $21,6^{\circ} \mathrm{C}$, while the real value is $21,3^{\circ} \mathrm{C}$ and the starting value is $20^{\circ} \mathrm{C}$. During this operative condition a limited low current discharge does not cause significant increases in temperature, however the problem appears after a continuous loop of several cycles.

The characterization of a virtual model requires also data such as heat capacity, cell density, and the superficial emissivity values. Even if the lithium-ion cells are constituted by several repetitive sub-layers, the material properties have been considered as average values over volume. Thus the simulation target is limited to analyze the average temperature distribution, in order to simplify the virtual model and to reduce the project lead time. While all the simulation parameters (such as those regarding the turbulence model), have been validated comparing the experimental data with the simulation results.

The implementation of virtual prototype methods allows the engineer to simulate different load profiles. In this test case the analysis related to the repetitive ECE R15 cycles has been described, but these methods can be extended to any customized driving cycles.

Figure 7 shows the cell temperature profile monitored by IR camera during the load related to defined driving cycle. While Figure 8 shows a report of virtual temperature distribution calculated by FVM solver, as a comparison with previous image (Figure 7Figure 8).

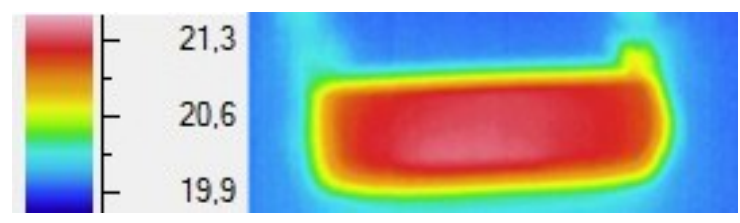

Figure 7 Temperature distribution monitored by IR camera at the end of test cycle

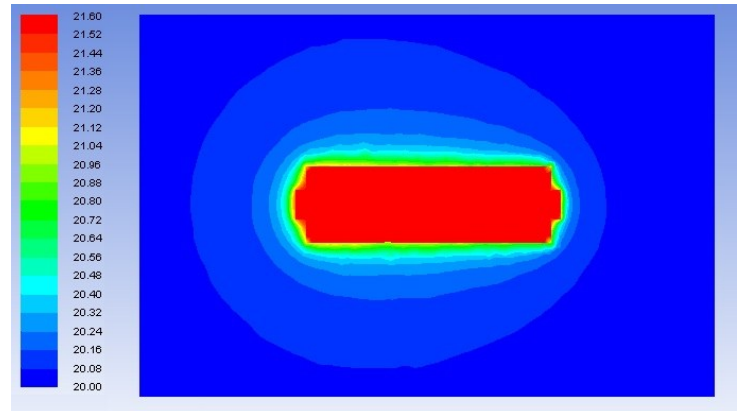

Figure 8 Temperature report of cell thermal simulation 


\subsection{Battery pack parameterization}

According to the explained methodology, the Battery Pack Layout Configuration concerns the definition of geometrical layout. This level provides 2D sections which are related to a library of 3D models. Some datasheets have been implemented to configure typical battery plant configurations. The engineer can reuse existing template models during design process or define a new model structure and add it in the related database. Each battery template model has to include the main dimensions such as cell size, elements distances, battery sizes and cells patterning. As example Figure 9 reports a parametric section of a small battery array with cylindrical elements. In this figure each dimension is represented by a parameter. The list of parameters becomes an input for the generation of simplified 3D model useful for virtual simulation. The aim of the geometrical modeling is the definition of a battery layout for cooling optimization. This method gives flexibility to the workflow platform in order to satisfy the SME needs.

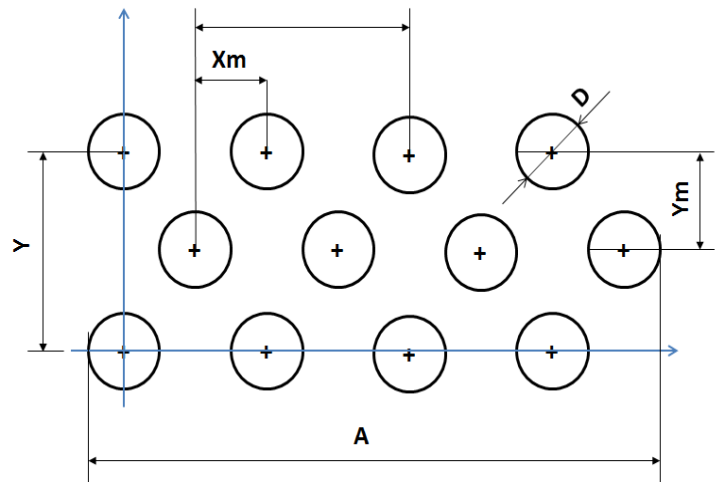

Figure 9: Example of parameterization for a small array with cylindrical cells

Generally a Li-ion battery is constituted by repetitive modules, and the virtual analysis only focus on the elementary cells array.

The layout shape concerns the cell arrangement into a battery pack or one repetitive module. A common used layout is that linear, where cells are ordered in parallel rows. While the offset layout regards a crossed arrangement of staggered rows (like a zigzag form) as shown in Figure 9. This offset structure is mostly used for cylindrical elements, while the parallel shape is common for the soft pouch type cells.

The cooling flow may be aspirated or pressurized, but generally is used the aspirated mode for electronic equipment like battery.

The cylindrical cells, used in the proposed test case, are suitable for an offset packaging. Thus an offset arrangement has been chosen with a cross air flow due to the difference between inlet and outlet into battery pack (Figure 10 and Figure 11).

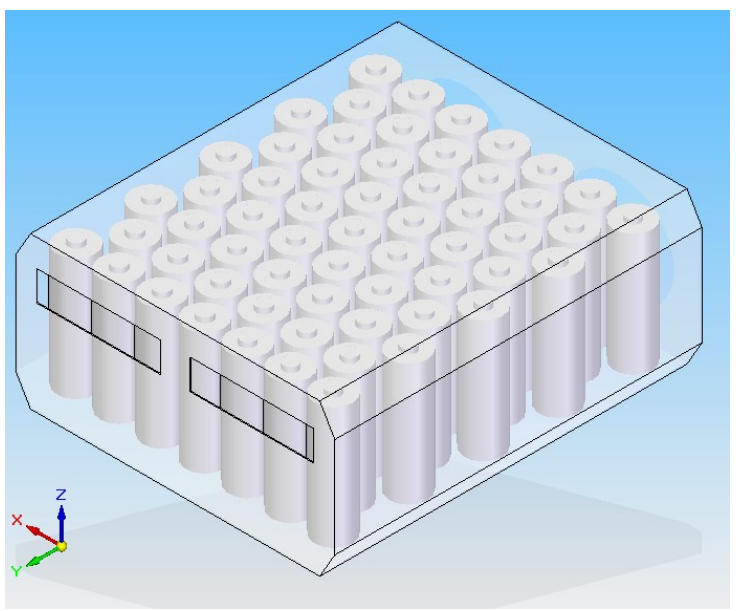

Figure $10 \mathrm{~A}$ frontal isometric view of analysed battery module with the two inlet sections

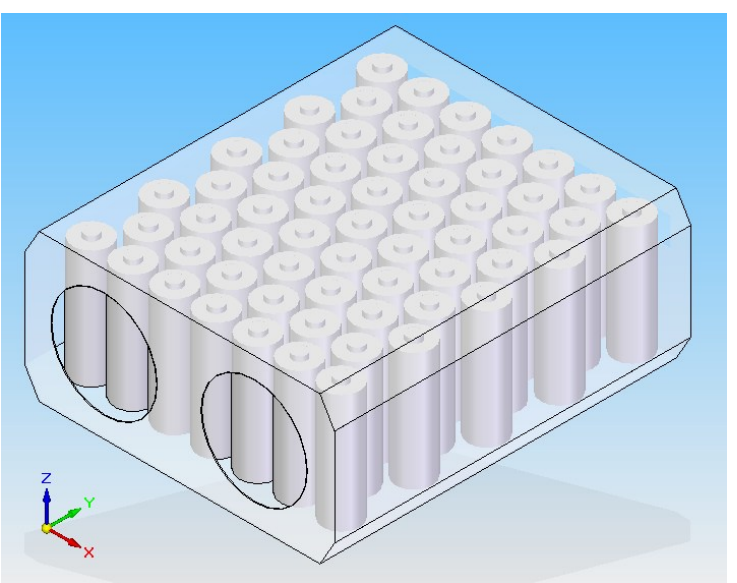

Figure 11 A rearward isometric view of analysed battery module with the two outlet sections

Following the proposed workflow, at this level the engineer can select battery layout and define main dimensions. In Figure 10 is shown the geometrical CAD model which has been configured for the test case using the Pack Layout Configuration tool. However, the geometrical details are manually modeled by the engineer which has to review the generated model. So, the configuration tool is an aid support, and this aspect lets to give flexibility to the virtual analysis process.

Next section discusses how the defined geometrical model becomes a virtual domain for the CFD simulation phase.

\subsection{Battery module simulation}

The second level of virtual prototyping regards the fluid dynamic simulation of a simplified battery model. Thus, at this section the CFD simulation 
regarding one of four identical battery module provided for the prototype hybrid vehicle, where one module contains 59 cells. Different thermo fluid dynamic parameters have been simulated, and a final configuration has been defined to provide a suitable temperature, and also a thermal uniformity during the working condition. The chassis of the battery pack is an important constraint in this test case. The space dimension is fixed and also the inflow and outflow sections have already been defined in order to apply this battery inside a specific vehicle frame.

The simulation domain analyzed concerns the symmetric part of virtual model. The use of symmetric feature allows to reduce the number of tetragonal element, generated during the mesh processing, and to limit also the computational load of un-steady simulations.

The CFD simulation reproduces the thermal behavior of a battery module (59 cells) under the operative condition of driving cycle, in order to evaluate the performance of air cooling. All the calculation is based on the analytical evaluation of the electrochemical heat source. This condition is an input for the FVM analysis.

Summary, the CFD simulations provide a means to estimate the temperature distribution in a battery pack module reproducing the operative condition in a virtual environmental.

Different geometrical layouts have been analyzed, but only one configuration has been reported (Figure 11). The air cooling is constituted of two compact fans (nominal diameter $100 \mathrm{~mm}$ ) that suck air from the battery interior and push it out through circular sections. The inlet section is arranged in the opposite side respect to the fan wheels.

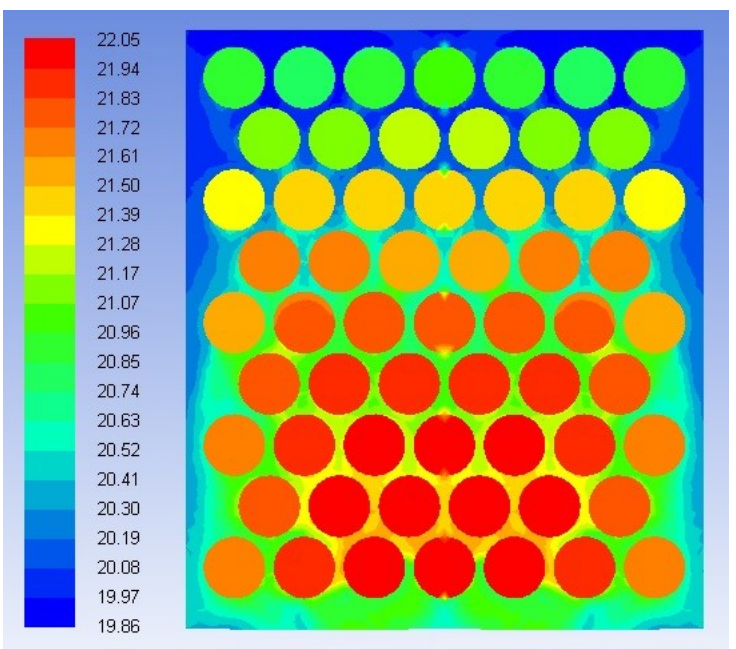

Figure 12 The temperature map on the middle section with air cooling at $20^{\circ} \mathrm{C}$ and $100 \mathrm{~m}^{3} / \mathrm{h}$ volumetric flow

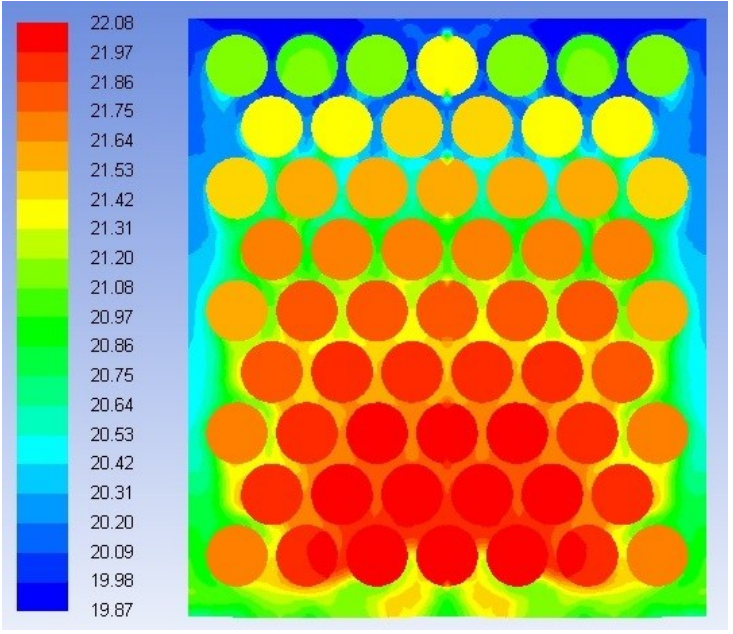

Figure 13 The temperature map on the middle section with air cooling at $20^{\circ} \mathrm{C}$ and $50 \mathrm{~m}^{3} / \mathrm{h}$ volumetric flow

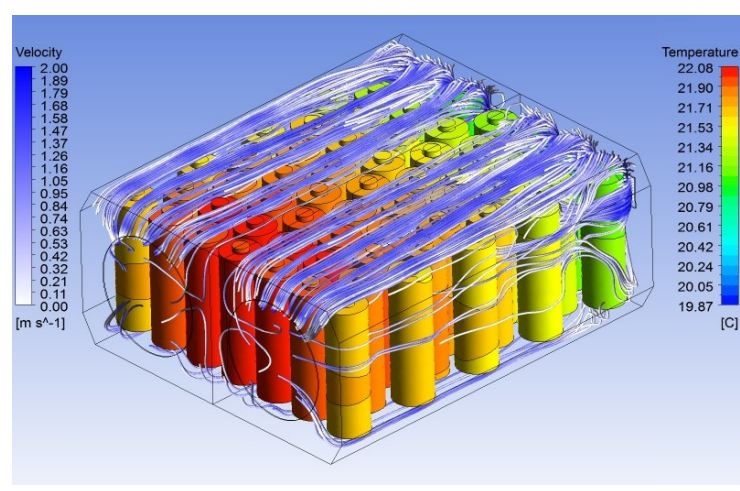

Figure 14 Isometric view of battery module including temperature map and velocity streamline for the cooling case of $50 \mathrm{~m}^{3} / \mathrm{h}$

Figure 12 shows the temperature profile related to the simulation with a volumetric flow of $100 \mathrm{~m}^{3} / \mathrm{h}$ sucked by two compact fans. This simulation reproduces the thermal load due to the cycle described above, constituted of 4 repeated ECE R15 driving cycles. In particular, the temperature distribution in Figure 12 regards the middle section of selected battery module. The starting temperature analyzed is $20^{\circ} \mathrm{C}$ and the air cooling flow is at the same temperature during the simulation. Using this setup, the maximum cell temperature, simulated after a driving cycle of 780 sec, is $22.05{ }^{\circ} \mathrm{C}$ (Figure 12), while the minimum value is $20.74{ }^{\circ} \mathrm{C}$ (with a gap of $1,31^{\circ} \mathrm{C}$ ).

On the other hand, in Figure 13 is shown the simulation report with a different air cooling condition. The intake air elaborated by compact fan wheels has a volumetric flow of $50 \mathrm{~m}^{3} / \mathrm{h}$. This second analysis aims to investigate the influence of air flow rate on the cooling performance, in order to evaluate the battery cooling with virtual prototyping tools. The maximum cell temperature, 
simulated for this second condition, is $22.08{ }^{\circ} \mathrm{C}$ (Figure 13), while the minimum value is 21.08 ${ }^{\circ} \mathrm{C}$ (with a gap of $1{ }^{\circ} \mathrm{C}$ ). Then in Figure 14 is shown an isometric view of the virtual model including both the temperature distribution over the cell skins and the velocity streamline. This representation is related to the volumetric flow of $50 \mathrm{~m}^{3} / \mathrm{h}$.

Comparing Figure 12 and Figure 13 the maximum values of temperature are almost the same, but the thermal distribution is different. In first case, with $100 \mathrm{~m}^{3} / \mathrm{h}$ volumetric airflow, it is shown a less average temperature. However this thermal difference is not really clear for several causes: the air velocity increasing introduces significant losses through the interstices between the cell elements, the cooling temperature is the same for both simulation, and in addition the analysis refers to a short test period of 13 minutes with low current rates during the simulated driving cycle. The final temperature achieved during simulation is higher than that one of the real test related to one cell (Figure 6). This difference is less than $1^{\circ} \mathrm{C}$, but the VP analysis presents a forced convection condition for air cooling, while the test on one cell has been conducted under natural convection. So the real temperature achieved by a single cell is less than the value reached inside a battery pack. This difference can be explained because in a battery the presence of several hot elements, packaged in a close way, limits the heat dissipation and favors the temperature increasing.

Anyway using virtual prototyping analysis the designer is able to valuate several settings and to investigate the causes of different thermal behaviors on parametric geometrical models.

In this case, the compact fan wheels selected for the described PHEV have been chosen comparing the thermo fluid dynamics behavior at different volumetric rate. The final choice has been to use a fan wheel type with variable volumetric flow, with $50 \mathrm{~m}^{3} / \mathrm{h}$ produced in the analyzed conditions.

Generally, the geometrical layout analyzed favors the heat exchange between the superficial cell skins and the air flow under the forced convection condition. An advantage related to this arrangement is the possibility to achieve a uniform temperature between each battery element.

\section{Conclusion}

In conclusion, the proposed approach shows a design methodology, based on virtual prototyping tools, which supports the engineer during the thermal analysis and the cooling design phase. The focus is on the cooling simulation of a PHEV Li-ion battery. As a test case, a battery pack with $10 \mathrm{Ah} \mathrm{LiFePO}_{4}$ cylindrical cells has been proposed.

The battery thermal analysis is based on the analytical calculation of heat generated by electrochemical reactions. This values have been used to reproduce the behavior of one cell in a FVM analysis, and to compare the temperature profile between real and virtual model. So, the first level of virtual analysis has regarded the simulation of one cell, but the second level has introduced the CFD computation of a complete battery module which contains 59 cells. The simulated thermal loads are related to an urban light vehicle. In particular the heat generated has been calculated by analytical formulation using the current profile related to the driving paths constituted by four repeated ECE R15 Urban Driving Cycles. Thus the FVM analysis have been conducted in unsteady condition.

A geometry configuration has been proposed for one battery module to obtain a suitable temperature and a cell thermal uniformity during the working condition. Then two simulation reports have been described with different air cooling flow rate. The results show a good thermal uniformity using the analyzed cooling settings.

In accordance to the needs of small and medium size companies, the research approach leads to a reduction in the cost and the project development lead-time. In this way the real pilot prototypes are replaced in part by virtual models.

For what concerns the research approach, a future development can be the introduction of BMS (battery management system) behavior during CFD simulation in order to consider different current profiles for each cell. In this case it will be also possible to simulate different thermal management algorithms during the design phase.

\section{Acknowledgments}

The authors wish to thank FAAM Group S.p.A. for their precious contribution in the development of this research program; particular acknowledgement to Eng. Roberto Isidori.

\section{References}

[1] S. Li, Automatic generation of assembly system configuration with equipment selection for automotive battery 
manufacturing, Journal of Manufacturing System, 30 (2011), 188-195

[2]

R. Raffaeli et al., Modular Product Configuration: An Automatic Tool for Eliciting Design Knowledge From Parametric CAD Models, Proceedings of the ASME IDETC/CIE 2010, Montreal, Quebec, Canada, DETC2010-28242

[3] C. Sen, Battery Pack Modeling for the Analysis of Battery Management System of a Hybrid Electric Vehicle, Vehicle Power and Propulsion Conference VPPC '09, ISBN 78-1-4244-2600-3, 2009

[4] S. Li, H. Wang, Y.T. Lin, J. Abell, S.J. $\mathrm{Hu}$, Benchmarking of high capacity battery module/pack design for automatic assembly system. Proceedings of the 2010 ASME International Manufacturing Science and Engineering Conference, 2010, p. 34114

[5] AA. Pesaran, Battery thermal models for hybrid vehicle simulations, Journal of Power Sources, 110(2), 377-82, 2002

[6] Z. Rao, S. Wang, A review of power battery thermal energy management, Renewable and Sustainable Energy Reviews, 15 (2011), 4554- 4571

[7] K.E. Thomas, J. Newman, Journal of the Electrochemical Society, 150 (2), A176A192, 2003

[8] Y. He, W. Liu, B.J. Koch, Battery algorithm verification and development using hardware-in-the-loop testing, Journal of Power Sources, 195 (2010), 2969-2974

[9] J. Randles, Kinetics of rapid electrode reactions, Discuss Faraday Soc., 1, 11-19, 1947

[10] M. Mousavi, S. Hoque, S. Rahnamayan, I Dincer, G.F. Naterer, Optimal Design of an Air-Cooling System for a Li-Ion Battery Pack in Electric Vehicles with a Genetic Algorithm, IEEE Congress on Evolutionary Computation (CEC), 2011

[11] D. Ghosh et al., Full Hybrid Electrical Vehicle Battery Pack System Design, CFD Simulation and Testing, Advanced Battery Technology 2010, ISBN 978-07680-3414-1, SAE International, 2010

[12] C. Forgez et al., Thermal modeling of a cylindrical LiFePO4/graphite lithium-ion battery, Journal of Power Sources, 195 (2), 2961-2968, 2010

\section{Authors}

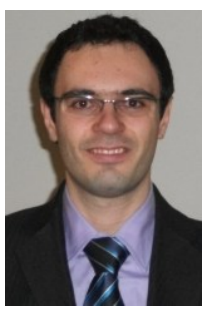

Dr. Paolo Cicconi,

$\mathrm{PhD}$. in Mechanical and Management Engineering at $\mathrm{PhD}$ School of Università Politecnica delle Marche; doctor thesis: Tools and Methods Based on Knowledge Elicitation to Engineering Design. $\mathrm{He}$ graduated in Mechanical Engineering and currently works research activities regarding Knowledge Management at Industrial Engineering and Mathematical Sciences Department of Polytechnic University of Marche.

Email: p.cicconi@univpm.it

PhD. Student Daniele Landi

is two years into his $\mathrm{PhD}$ studies at the Università Politecnica delle Marche. Currently he is working on methods and tools for virtual prototyping in the Industrial Engineering and Mathematical Sciences Department of the Polytechnic University of the Marche. He graduated in Mechanical Engineering with a thesis entitled: "Study of methods for virtual prototyping".

Email: d.landi@univpm.it

Prof. Michele Germani.

is Associate Professor at the Università Politecnica delle Marche, Department of Industrial Engineering and Mathematical Sciences. His main research topics are: eco-design methods and tools, advanced virtual prototyping tools,

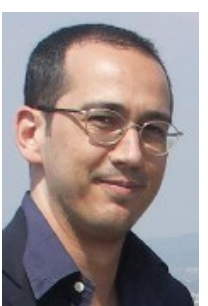
energy efficiency and humanmachine interaction. He has written 150 scientific papers published in international journals and conference proceedings and he is responsible for several European and National research projects.

Email:m.germani@univpm.it 